\title{
Exploring Patient Engagement: a qualitative analysis of low- income urban participants in asthma research
}

\author{
Amy Korwin, MD ${ }^{1}$, Heather Black, PhD ${ }^{2,3}$, Luzmercy Perez, BA ${ }^{2,3}$, Knashawn H. Morales, \\ $\mathbf{S c D}^{3,4}$, Heather Klusaritz, PhD, MSW ${ }^{3,5}$, Xiaoyan Han, $\mathbf{M S}^{3,4}$, Jingru Huang, BA ${ }^{2,3}$, Marisa \\ Rogers, MD, MPH ${ }^{1,3}$, Grace Ndicu, AS ${ }^{2,3}$, and Andrea J. Apter, MD, MSc, MA ${ }^{1,2,3}$ \\ ${ }^{1}$ Hospital of the University of Pennsylvania, Department of Medicine \\ 2Division of Pulmonary Allergy Critical Care Medicine \\ ${ }^{3}$ Perelman School of Medicine, University of Pennsylvania \\ ${ }^{4}$ Department of Biostatistics and Epidemiology \\ ${ }^{5}$ Department of Family Medicine and Community Health
}

\begin{abstract}
Background-Uncontrolled asthma is a common highly-morbid condition with worse outcomes in low-income and minority patients in part due to barriers accessing and engaging with healthcare. We developed a patient advocate to educate about and assist with navigating access to care and provider-patient communication. Participants completed an End of Study Questionnaire (ESQ) which was analyzed to assess experience and engagement with the protocol.
\end{abstract}

Objective-This study uses qualitative analysis to evaluate participant experience with the Patient Advocate and control group interventions.

Methods-The ESQ aimed to prompt open-ended discussion of study experience. Questions were developed from patient focus groups about the patient advocate intervention (PAI), and were revised based on early responses. The questionnaire was administered after 12 months of study participation: 6 months of control or PAI, followed by 6-months of follow-up. Answers were evaluated using qualitative coding and a Grounded Theory analytical approach.

Results-102 low-income and minority adults with moderate or severe asthma who have completed the study protocol at the time of publication (approximately one-third of total participants) found PAI and control group activities acceptable. Four themes emerged from both groups: 1) appreciation of interpersonal and educational interaction, 2) perception of improved healthcare adherence, 3) preparedness for physician appointments, 4) improved patient-provider communication. Attention from study personnel and review of asthma-related information was unanimously well-received and empowered patients' active healthcare participation.

Corresponding author: Andrea J. Apter, MD, MSc, MA, 829 Gates Building, Hospital of the University of Pennsylvania, 3600 Spruce Street, Philadelphia, PA 19104. Telephone: (215) 662-2775, FAX : (215) 615-5055, Andrea.Apter@uphs.upenn.edu.

Publisher's Disclaimer: This is a PDF file of an unedited manuscript that has been accepted for publication. As a service to our customers we are providing this early version of the manuscript. The manuscript will undergo copyediting, typesetting, and review of the resulting proof before it is published in its final citable form. Please note that during the production process errors may be discovered which could affect the content, and all legal disclaimers that apply to the journal pertain. 
Conclusion-Patient engagement and empowerment were elicited by perceived education and personal attention. This study suggests a low-resource, feasible method to improve patient engagement.

\section{Keywords}

asthma; patient advocate; patient engagement; access to healthcare; health disparities; adherence

\section{Introduction}

Asthma is a chronic, costly, and often debilitating medical condition that affects more than 25 million people in the $\mathrm{US}^{1}$ and results in significant healthcare utilization: over 10 million outpatient appointments ${ }^{2}, 1.5$ million emergency department visits, and 400,000 hospitalizations ${ }^{3}$, costing over $\$ 30$ billion $^{4}$ annually. It is well-described that worse health outcomes are seen in low-income and minority patients. African American patients with asthma have double the risk of death related to this condition, and more than twice the number of hospitalizations and emergency department visits as compared with white patients. Similar disparities are seen in Puerto Rican patients ${ }^{1}$.

These trends are likely multifactorial, secondary to environmental factors, such as allergen and particulate matter exposures, as well as barriers to accessing and engaging with teams of healthcare providers ${ }^{3}$. Recent efforts have attempted to improve patient engagement, yet significant challenges exist. Patients may be impeded by poor health literacy or overall limited education, may not feel empowered to participate ${ }^{6}$, or may be uncomfortable fully discussing their concerns in a short clinical appointment. Provider factors may also impede effective communication, including professional pressures such as brief appointments and distracting alerts on electronic medical records, and personal characteristics such as lack of empathy.

The majority of studies investigating approaches to improving asthma outcomes in this population have proposed either educational interventions targeting patients, or systemslevel interventions targeting providers ${ }^{1}$. We adapted the patient navigator initially proposed by Harold Freeman, et al ${ }^{7,8}$ and developed a patient advocate program that introduces a new clinical role fulfilled by a young college graduate, namely the patient advocate (PA), with the goal of helping patients to successfully navigate access to care and facilitate providerpatient communication. Initial results demonstrated acceptability, feasibility, and positive patient response to the patient advocate pilot ${ }^{9}$, which prompted continuation of this project with more participants and longer follow up. As part of this ongoing effort an End of Study Questionnaire (ESQ) was developed to further investigate participants' experiences with the study. This investigation aimed to evaluate patients' experiences with both the control and intervention arms of this study, with particular focus on a sense of patient empowerment and engagement as well as acceptability of this protocol and thus sustainability of intervention. 


\section{Methods}

\section{Study Design}

The ESQ was incorporated into the ongoing Helping Asthma Patients 2 (HAP2) Study. This study is a randomized controlled clinical trial that investigates the effects of a patient advocate intervention (PAI) on asthma outcomes in low-income or minority patients with moderate-to-severe asthma. A total of 312 patients were recruited from 8 clinics serving low-income inner city neighborhoods, and were randomized $1: 1$, stratified by practice, to either receive the PA intervention or control for 6 months, followed by 6 months of observation.

Research coordinators: Patient Advocates and Data Collectors-A research coordinator acted as either a PA or a data collector for any individual patient. Research coordinators were college graduates interested in healthcare careers. They were trained in asthma education, patient interaction, research principles, protocol integrity, cultural competence, and spirometry; but no other medical tasks. Participants randomized to control received routine medical care from their providers and had contact with a data collector during the intervention period; participants randomized to PAI had contact with both a data collector and their assigned PA. Each participant randomized to PAI was assigned a PA who followed him or her longitudinally. Primarily Spanish-speaking participants had PA's who could communicate effectively in Spanish. The PA responsibilities included assisting with appointment reminders, clinic visit preparation and agenda, and administrative tasks to navigate healthcare. They attended appointments with the participant and took notes, and ensured that the participant could "teach back" important information from the visit. Data collection visits occurred every 3 months with all participants in both groups; these included asking questions about knowledge of inhaled corticosteroids, inhaler technique, and basic asthma facts. Incorrect answers on the Inhaled Corticosteroid Knowledge Questionnaire (Table E-1 in the Online Repository) and the Inhaler Techniques Questionnaire (Table E-2 in the Online Repository) were reviewed and corrected for patients in both control and PAI groups.

End of Study Questionnaire-The ESQ (Table 1) was administered at the final data collection visit, designed to occur 12 months after initial recruitment and randomization. The ESQ is comprised of nine items and aimed to prompt participants to discuss their experience with HAP2 in an open-ended manner. Questions were developed initially from patient responses to questions about PAs in focus groups from which the PA role was developed ${ }^{10}$. Later the questions were revised based upon participant responses, with additional questions added regarding visit preparation, changes in communication from doctor to patient, and changes in communication from patient to family and friends ${ }^{11,12}$. Thirty three participants received the first version of the ESQ, and 69 participants received the updated version; thus only the latter group of participants answered the three questions referenced above. Participants were asked to rate their perceptions of various aspects of the study and to qualitatively describe their reasoning. 


\section{Participants}

Participants in both arms of the HAP2 study were the respondents to this questionnaire. Inclusion criteria included age 18 years or older with physician's diagnosis of asthma and prescription of either inhaled corticosteroid and long-acting bronchodilator combination therapy ( $n=92)$, medium dose inhaled corticosteroid $(n=6)$, or otherwise met NHLBI Guideline criteria for moderate or severe persistent asthma including reduced FEV1 ( $n=4)$. All participants had spirometry showing evidence of reversible airflow obstruction with (a) forced expiratory volume in one second $(\mathrm{FEV} 1)<80 \%$ predicted at the time of screening or within 3 years prior, and (b) improvement with bronchodilator: either (i) an increase of at least $15 \%$ and 200ml in FEV1 with asthma treatment over the previous 3 years, or (ii) an increase in FEV1 or FVC > 12\% and improvement in FEV1 by at least 200ml within 30 minutes following 4 puffs of albuterol by MDI (or $2.5 \mathrm{mg}$ by nebulizer). The participants also had at least one appointment scheduled with the clinician managing their asthma within the first six months of the study; the number of appointments scheduled was at the discretion of asthma provider, which varied. Sometimes a patient was seen by both a general practitioner and an asthma specialist. Participants were excluded if they had significant psychiatric or cognitive comorbidities, were unable to provide informed consent, could not communicate in English or Spanish, or had participated in the HAP pilot study. All HAP2 study subjects who completed the final data collection visit participated in ESQ data collection.

\section{Procedures}

The ESQ was administered to all participants during the final data collection visit, designed to occur 12 months after recruitment and randomization. Data collectors verbally asked the items on the questionnaire and recorded the participant's response verbatim. Participants were asked to provide numeric ratings and responses to open-ended questions, to which they were able to respond minimally or extensively. What follows is an analysis of all open-ended responses at the point saturation of concepts was achieved.

\section{Analysis}

The data obtained in the ESQ responses were coded and analyzed for dominant themes using the Grounded Theory approach ${ }^{13}$. Responses were first compiled into an excel database and then analyzed by the study team to recognize emerging themes. Answers to each question were coded according to overall tone (positive, negative, or neutral), and presence or absence of mentioning dominant themes that were identified during team meetings. Two members of the study team who were not directly involved in administration of the ESQ separately coded. Data were analyzed for qualitative difference in dominant themes between PAI and control, as well as overarching themes shared between both groups.

\section{Results}

Of the HAP2 participants ( $\mathrm{n}=312), 102$ had completed the final data collection visit and the ESQ at the time of saturation. The 102 participants had similar demographics in both groups (Table 2). Notably there was no significant difference between groups in scores on baseline assessments measuring Asthma Quality of Life, Asthma Control, numeracy, and reading comprehension. The Asthma Quality of Life mean score is low compared to the original 
population in which the questionnaire was validated. Seventy-four (73\%) participants had an Asthma Control Questionnaire score in the uncontrolled range $(>1.5)$. The mean score at baseline was $2.4+1.3$. The numeracy baseline score showed a mean of fewer than 2 out of 4 questions correct with 64 participants (63\%) having a score $<=2$. Seventy-five $(74 \%)$ had a reading comprehension score (Short Test of Functional Health Literacy in Adults (See Table 2)) in the adequate range ${ }^{30}$. These metrics indicate that the study population had limited health literacy and at the time of enrollment had poor control of asthma with symptoms significantly reducing quality of life. No differences were noted in responses between male and female participants.

Overall, four prominent positive themes emerged from responses to the ESQ reflecting participants' experiences with the HAP2 study: 1) appreciation of an interpersonal and educational intervention, 2) subjective perception of improved healthcare adherence, namely better medication compliance and provider follow up, 3) preparedness for physician appointments, and 4) improved patient-provider communication. Participants in the PAI group more frequently commented on increased use of notes to prepare for appointments and improved communication with friends and family as compared with control group; otherwise all trends occurred in both groups.

\section{Interpersonal and educational intervention was universally appreciated}

Across both control and PAI groups, all participants reported a positive overall experience with the study, and every person said they would recommend this program to a peer with asthma. When asked to rate overall satisfaction with the study on a scale from $1-5$,with 5 indicating great satisfaction, all but five participants rated their experience 5; four of the remaining participants rated 4 and one participant did not respond. Open-ended responses accompanying ratings less than 5 did not qualitatively differ from those with superior rating, and reflected positive experiences with the study: one commented that it was informative, one commented on improved medication and general healthcare adherence, and two appreciated the personal interaction with study personnel. Participants in both groups most commonly cited perceived learning as the reason for their positive experience (control 31/54; PAI 27/48).

- "The study was good because it kept me focused on doing the right things. Since I have so many health issues, when something is not actively bothering me, I don't think about it as much. I try to keep things dusted and take my medicines as I should. The study reminds me that I don't know everything about asthma. It keeps me up to date since things are constantly changing, medicines are changing." -PAI group

- $\quad$ "I learned more about asthma and it kept me on my toes with taking my medications and knowing which medications I am on." -Control group

Participants commented specifically on education related to their medications, with improved understanding of mechanism of action, differences between inhalers, and appropriate inhaler technique. 
- "It gave me more insight on my medicines and what they do and how they help my asthma." -Control group

- $\quad$ "It reinforced my understanding of the differences between medication dosages and uses. I learned more about my particular asthma and its complications." Control group

- "[I learned] about the use of inhalers. I was a person that before I was taking the steroids for symptoms instead of albuterol. Now I know to use the albuterol as a rescue inhaler." - Control group

Participants' positive experience was further evident in their universal response that they would recommend study participation to peers with asthma. The most commonly cited reason was perceived educational benefit. (19/54 Control, 19/48 PAI). Quotations in response to being asked if they would recommend this study to a peer with asthma:

- $\quad$ "Yes, so they can get informed and learn about asthma and how to properly take their medications." - PAI group

- $\quad$ "Yes, because it's helpful by reinforcing what your doctor says and picks up what your doctor may have failed to mention." - Control group

Participants in both groups commented on interactions with study personnel as contributing to their satisfaction.

- " "[The data collector] was very accommodating and delightful to be with during my visits." - Control group

- $\quad$ "I liked the questions and company. [...] I benefitted by talking about asthma and getting it under control." - PAI group

\section{Improved healthcare adherence}

Participants randomized to both control and PAI groups felt that their asthma medication adherence improved as a result study participation. This trend was similarly represented in both study groups. (Q3, Adherence: 14/54 Control, 8/48 PAI)

- I am more concerned about my health and asthma. I am more aware of taking meds." -Control group

- "The study makes you aware of how important your medicines are, [...] and how important it is to take your medicines on time." - PAI group

Some participants commented that this experience improved adherence in other areas of healthcare. (Q3, Health in general: 14/54 Control, 8/48 PAI)

- $\quad$ "It made me more compliant and kept me aware of other medications (nonasthma meds)." - Control group

- $\quad$ "I now know I have to stay on top of my appointments and not miss them. Before, I was canceling my appointments but not anymore.” - PAI group 
Many participants noted that their perceived improvement in adherence was due to an increased awareness of potential health consequences associated with asthma. (Q3, Awareness: 7/54 Control, 10/48 PAI; Q7, Awareness of asthma: 15/54 Control, 9/48 PAI)

- II've always been concerned about my health, but [the study made me] more concerned about my asthma and taking care of myself." - Control group

- I take my asthma more seriously. And I try to remember to take my medications. Before I didn't really care.” - PAI group

\section{Improved physician appointment preparation}

Participants in both arms of the study felt they could more effectively prepare for physician appointments as a result of HAP2. Participants in the PAI group were more likely than patients in the control group to mention improved visit preparation when prompted openendedly to discuss what they learned in this study, while participants in both groups were similarly likely to endorse a change in physician appointment preparation when asked about it specifically. Several participants commented about learning to prepare questions or bring additional information to the office visit, such as medication lists or recounting recent flares. (Q3: Preparation for appointments: 3/54 Control, 7/48 PAI; Q4: Change in visit prep endorsed by 19/42 Control, 13/27 PAI).

- "I learned to always be on time for appointments and to ask questions when I do come in for appointments." - PAI group

- $\quad$ "I ask more questions about my medications and discuss how my medications help improve my asthma." - Control group

Participants in the PA group were more likely to comment on use of written notes to facilitate appointment preparedness.

- $\quad$ "I am going to keep a journal now. My medical visits want me to know when I have taken prednisone; help record any appointments and flare-ups." - PAI group

- $\quad$ "I will make sure I have notes with me, to write down if something changes, to ask questions, to prepare by writing those questions down." - PAI group

\section{Improved communication}

Participants in both PAI and control groups noted a change in communication with their physician following their study experience (14/54 Control, 14/48 PAI). Regarding change in communication with physicians, participants from both groups most commonly commented that they were more open.

- "The study changed how my doctor talks to me during visits because since I ask questions, he explains things more now." - PAI group

- $\quad$ "Since I was more forthcoming, so was my doctor. He spent more time with me. I found the communication flowed a lot better and was more in depth, maybe because I was more open." - PAI group 
- "I learned how to ask more questions than I usually do. I make sure [my doctor] answers so that I understand them." - Control group

- " I've learned to be more candid and really express how I feel." - Control group

- $\quad$ "The study taught me to always ask my doctor when I have questions. Sometimes I had been afraid to ask questions and wouldn't understand why I couldn't take certain medicines." - PAI group

Participants in the PAI group were more likely to comment on change in communication with family and friends, with the most common responses indicating more open or informative conversation (Q8: Change w/ family/friends endorsed by 13/42 Control, 12/27 PAI).

- $\quad$ "I used to be more reserved when talking about my health issues but now I talk more freely about my problems and what the doctor says" - PAI group

- "The study gave me a lot of information to pass on to my family and friends." PAI group

\section{Discussion}

The HAP2's ESQ provides insight into participants' experiences with both the PAI and the control group. At completion, participants universally responded that they would recommend this study to a friend with asthma, providing evidence of their support ${ }^{14}$. Participants from both groups appreciated an educational and interpersonal intervention, and felt that the study improved healthcare and medication adherence, visit preparation, and communication.

Taken together, these themes suggest that the participants' experiences in this study made them feel more empowered to take control of and to fully engage in their healthcare. This was true in our study whether the participant was randomized to the PAI or to the control group. By study design, the PAs fulfilled a unique role whose responsibilities are not systematically addressed by current healthcare personnel. They provided structured informative encounters and acted as a personal contact to help navigate the health system. Data collectors administered study questionnaires to all participants in both groups and provided feedback on incorrect answers. Thus participants in the control group received additional communication, individual contact, and information from the study personnel as compared with baseline patient experience. These changes, while not the designed study intervention, were sufficient to elicit positive responses and improve patient engagement.

A positive effect from study participation itself has been well-described in several study designs within healthcare research, including with self-reported data, further validating the benefit of personal attention and accountability suggested by our results ${ }^{15,16}$. Thus, facilitating engagement in the study may depend on making a meaningful experience for participants in the control arm as well as in the intervention. Thus in the future, the comparison in such research should potentially be considered an active one with a control group, rather than considered true "usual care." 
With the advent of care models focusing on patient involvement in healthcare, such as Patient Centered Care and Shared Decision Making, active patient engagement has become very important in delivering effective care. As defined by Hibbard et al, patient activation is "understanding one's role in the care process and having the knowledge, skill and confidence to manage one's health and health care ${ }^{17}$." Philosophically, increasing patient involvement in care is consistent with the ethical principles of autonomy and agency ${ }^{18}$; medically, it has been shown to correlate with improved health outcomes including adherence with asthma treatment ${ }^{19}$ and better overall health status ${ }^{20}$, among several others. In a meta-analysis to investigate efficacy and feasibility of interventions to improve patient engagement, Grande et al found that methods which inform and prompt patient involvement are more likely to effect a significant change in behaviors around healthcare when also addressing communication ${ }^{21}$. This is particularly important in minority patients, whose engagement may be more inhibited by a larger perceived power differential between themselves and the provider. Routine adoption of such interventions has not yet occurred in clinical practice; implementation is limited by a high learning burden for the patient, excessive resources or workflow alteration imposed on the providers, or both ${ }^{21}$. Thus, identifying an effective and feasible method for improving patient activation remains an important target for improving health outcomes.

The ESQ responses suggest that patient engagement and a sense of empowerment can be elicited by perceived education and personal attention. An intervention requiring minimal resources and time can achieve these goals. In this study we show that appropriately trained college graduates administering questionnaires and providing feedback on incorrect answers was received positively by patients and resulted in empowerment and active participation in healthcare, as subjectively reported. Participants were not overwhelmed by the learning burden, and the study design ensured that provider workflow was not affected. This study raises the exciting possibility of a low-resource, easily-implemented method to reliably improve patient engagement with healthcare.

Strategies to improve patient engagement must also effect change in provider factors. One question on the ESQ asked patients to report any perceived difference in communication from providers, and the overwhelming majority of respondents in both groups did not notice a difference. This study was mainly designed to investigate the patient response to the study protocol, rather than its impact on the provider. While outside the scope of the current discussion, the topic of changes in provider behavior remains an interesting and important area for further investigation.

Limitations of the ESQ and analysis include the use of open ended questions (which meant that not all participants were answering questions in the same way), the possibility of social desirability bias (participants answering in a way that they believe would be acceptable to the interviewer), and generalizability to a broader patient population. Additionally, given the qualitative nature of examining acceptability and engagement in this ongoing investigation, results are not associated with measures of health outcomes. While open ended questions allow patients to describe their thoughts and opinions more fully and allow for a diversity of experiences to be expressed, they do not require that patients all use the same framework to respond to a question. This limits the ability to conduct categorical tests of difference in 
responses among groups, and relies on the ability and willingness of participants to articulate their thoughts. A social desirability bias may have been introduced, particularly to the discreet numerical scale responses, by our method of using study personnel (namely data collectors) to administer the questionnaire. Finally, our study focused on a specific population of patients with moderate to severe asthma and low-income or minority status, limiting our ability to demonstrate that these results would apply to a general population. Taking these limitations into account, the existing data still present robust themes as discussed above to describe this particular study and provides new directional information for further exploration in future studies.

Several areas of future study are suggested by these results. As more data is collected, the potential for superior patient engagement strategies and empowerment in the PAI as compared to the control group should be investigated, and the impact of the control group as its own intervention should be quantified. Accompanying quantitative analysis should be pursued to investigate effects of this intervention on health outcomes. Specifically, research should be conducted to confirm subjective patient reported outcomes, such as asthma knowledge, medication adherence and follow up, and health outcomes related to morbidity in asthma compared between study groups. Feasibility and cost effectiveness data should also be obtained to investigate the logistics of incorporating this type of intervention into clinical practice. Future studies might include different modes of questionnaire administration to reduce social desirability bias, such as online survey or third party involvement. Finally, implementing a PA program with a more numerous and diverse patient population would provide more generalizable results.

\section{Supplementary Material}

Refer to Web version on PubMed Central for supplementary material.

\section{Acknowledgments}

Funding Source: This project was funded by NIH/NHLBI R18 HL116285

\section{Abbreviations}

PA patient advocate

ESQ End of Study Questionnaire

HAP2 Helping Asthma Patients 2 Study

PAI patient advocate intervention

FEV1 forced expiratory volume in one second

FVC forced vital capacity

MDI metered dose inhaler 


\section{References}

1. Akinbami LJ, Moorman JE, Bailey C, Zahran HS, et al. Trends in Asthma Prevalence, Health Care Use, and Morality in the United States, 2001-2010. NCHS Data Brief No.94. May.2012:1-8.

2. Press VG, Pappalardo AA, Conwell MD, Pincavage AT, Pochaska MH, Arora VM. Interventions to Improve Outcomes for Minority Adults with Asthma: A Systematic Review. J Gen Intern Med. 27(8):1001-15.

3. Cherry, DK., Hing, E., Woodwell, DA., Rechtsteiner, EA. National Ambulatory Med Care Survey: 2006 summary. Hyattsville, MD: National Center for Health Statistics; 2008 Aug 6. p. 1-39.

4. [Accessed April 24, 2016] CDC National Asthma Control Program Asthma Fast Facts. Available at:http://www.cdc.gov/asthma/pdfs/asthma_fast_facts_statistics.pdf

5. Canino G, McQuaid EL, Rand CS. Addressing asthma health disparities: A multilevel challenge. J Allergy Clin Immunol. 2009; 123:1209-1217. [PubMed: 19447484]

6. Izarry T, DeVito Dabbs A, Curran CR. Patient Portals and Patient Engagement: A State of the Science Review. J Med Internet Res. 2015 Jun 23.17(6):e148. [PubMed: 26104044]

7. Freeman HP. Patient navigation: a community centered approach to reducing cancer mortality. J Cancer Educ. 2006; 21:S11-S4. [PubMed: 17020496]

8. Freeman HP. A model patient navigator program. Oncol Issues. 2004; 19:44-6.

9. Apter AJ, Wan F, Reisine S, Bogen DK, Rand C, Bender B, Bennett IM, Gonzalez R, Priolo C, Sonnad SS, Bryant-Stephens T, Ferguson M, Boyd RC, Have TT, Roy J. Feasibility, acceptability, and preliminary effectiveness of patient advocates for improving asthma outcomes in adults. Journal of Asthma. Jul 17; 2013 50(8):850-860. [PubMed: 23800333]

10. Black HL, Priolo C, Akinyemi D, et al. Clearing clinical barriers: enhancing social support using a patient navigator for asthma care. J Asthma. 2010; 47:913-9. [PubMed: 20846085]

11. Black HL, Priolo C, Gonzalez R, Geer S, Adam B, Apter AJ. An analysis of contextual information relevant to medical care unexpectedly volunteered to researchers by asthma patients. $\mathrm{J}$ Asthma. 2012; 49:731-7. [PubMed: 22788543]

12. Apter AJ, Wan F, Reisine S, et al. Feasibility, acceptability and preliminary effectiveness of patient advocates for improving asthma outcomes in adults. J Asthma. 2013; 50:850-60. [PubMed: 23800333]

13. Straus, A., Corbin, J. Basics of qualitative research: Grounded theory, procedures, and techniques. Sage Publications; Newbury Park, CA: 1990.

14. Hamilton DF, Lane JV, Gaston P, Patton JT, Macdonald DJ, Simpson AH, Howie CR. Assessing treatment outcomes using a single question: the net promoter score. Bone Joint J. 2014 May; 96B(5):622-8. [PubMed: 24788496]

15. McCambridge J, Kypri K, Elbourne D. Research participation effects: a skeleton in the methodological cupboard. J Clin Epidemiol. 2014 Aug; 67(8):845-9. [PubMed: 24766858]

16. McCambridge J, Witton J, Elbourne DR. Systematic review of the Hawthorne effect: new concepts are needed to study research participation effects. J Clin Epidemiol. 2014 Mar; 67(3):267-77. [PubMed: 24275499]

17. Hibbard JH, Greene J. What the evidence shows about patient activation: better health outcomes and care experiences; fewer data on costs. Health Aff. 2013; 32:207-14.

18. Moulton B, King JS. Aligning ethics with medical decision-making: the quest for informed patient choice. J Law Med Ethics. 2010; 38:85-97. [PubMed: 20446987]

19. Wilson SR, Strub P, Buist AS, Knowles SB, Lavori PW, Lapidus J, et al. BOAT Study Group. Shared treatment decision making improves adherence and outcomes in poorly controlled asthma. Am J Respir Crit Care Med. 2010; 181:566-77. [PubMed: 20019345]

20. Hasskard Zolnierek KB, Dimatteo MR. Physician communication and patient adherence to treatment: a meta-analysis. Med Care. 2009; 47:826-34. [PubMed: 19584762]

21. Grande SW, Faber MJ, Durand MA, Thompson R, Elwyn GA. classification model of patient engagement methods and assessment of their feasibility in real-world settings. Patient Education and Counseling. 2014; 95:281-287. [PubMed: 24582473] 
22. Juniper EF, Buist AS, Cox FM, Ferrie PJ, King DR. Validation of a standardized version of the Asthma Quality of Life Questionnaire. Chest. 1999; 115(5):1265-1270. [PubMed: 10334138]

23. Juniper EF, Guyatt GH, Cox FM, Ferrie PJ, King DR. Development and validation of the Mini Asthma Quality of Life Questionnaire. The European respiratory journal. 1999; 14(1):32-38. [PubMed: 10489826]

24. Juniper EF, Guyatt GH, Willan A, Griffith LE. Determining a minimal important change in a disease-specific Quality of Life Questionnaire. J Clin Epidemiol. 1994; 47(1):81-87. [PubMed: 8283197]

25. Juniper EF, O’Byrne PM, Ferrie PJ, King DR, Roberts JN. Measuring asthma control. Clinic questionnaire or daily diary? Am J Respir Crit Care Med. 2000; 162(4 Pt 1):1330-1334. [PubMed: 11029340]

26. Juniper EF, O’Byrne PM, Guyatt GH, Ferrie PJ, King DR. Development and validation of a questionnaire to measure asthma control. The European respiratory journal. 1999; 14(4):902-907. [PubMed: 10573240]

27. Juniper EF, O'Byrne PM, Roberts JN. Measuring asthma control in group studies: do we need airway calibre and rescue beta2-agonist use? Respir Med. 2001; 95(5):319-323. [PubMed: 11392570]

28. Juniper EF, Bousquet J, Abetz L, Bateman ED, Committee G. Identifying 'well-controlled' and 'not well-controlled' asthma using the Asthma Control Questionnaire. Respir Med. 2006; 100(4): 616-621. [PubMed: 16226443]

29. Apter AJ, Cheng J, Small D, et al. Asthma numeracy skill and health literacy. The Journal of asthma : official journal of the Association for the Care of Asthma. 2006; 43(9):705-710. [PubMed: 17092853]

30. Baker DW, Williams MV, Parker RM, Gazmararian JA, Nurss J. Development of a brief test to measure functional health literacy. Patient Educ Couns. 1999; 38(1):33-42. [PubMed: 14528569] 


\section{Clinical Highlights Box}

1. What is already known about this topic? It is well-described that worse health outcomes are seen in low-income and minority patients. Common challenges to patient engagement include poor health literacy or limited education, or a limited sense of empowerment to participate.

2. What does this article add to our knowledge? This investigation demonstrates that patients who receive personal attention and a brief educational intervention feel empowered to be involved and more proactive in their healthcare, in both clinic and home settings.

3. How does this study impact current management guidelines? This study suggests a low-resource, feasible method to improve patient engagement with perceived education and personal attention. 


\section{Table 1}

\section{End of Study Questionnaire, Final Version}

1) Please tell me about your experience with the study overall on a scale from 1 to 5 , where 1 is "terrible experience" and 5 is "great experience."

(terrible experience) $1120344 \quad 5 \quad$ (great experience)

Probes:

- To what extent was participating in this study a good experience for you? Please tell me more about what was good for you.

- $\quad$ To what extent were participating in the study NOT a good experience for you? Please tell me more about what was NOT good for you.

2) For those who were randomized to PA only. For you personally, how helpful was the PA program overall on a scale from 1 to 5 where 1 is not helpful at all and 5 is very helpful.

(not helpful at all) $11203 \quad 4 \quad 5 \quad$ (very helpful)

Probes:

- $\quad$ Please tell me more about why you gave your experience with the PA program that rating.

- What did your PA do with you / for you?

- What were some of the positives and what were some of the negatives that you experienced?

- What else could the PA do with patients before the visit? During the visit? After the visit?

3) To what extent did you learn new things about asthma by being in the study? How much did you learn on a scale from 1 to 5 where 1 is "learned nothing new" and 5 is "learned many new things."

(nothing new) $12 \quad 2 \quad 3 \quad 4 \quad 5 \quad$ (many new things)

Probe (for those with any rating over 1): Tell me more about what you learned that was new.

4) To what extent did your participation in the study change how you will prepare for a visit in the future? Please tell me more about that.

5) To what extent did your participation in the study change how you talk to your doctor during a visit? Please tell me more about that.

6) To what extent did your participation in the study change how your doctor talked to you during a visit? Please tell me more about that.

7) To what extent did your participation in the study change how you think about your health? Please tell me more about that.

8) To what extent did your participation in the study change how you talk with your family and friends about your health? Please tell me more about that.

9) If you had a friend with asthma, would you recommend the study to your friend?

J Allergy Clin Immunol Pract. Author manuscript; available in PMC 2018 November 01. 
Table 2

Participant baseline characteristics by group assignment.

\begin{tabular}{|c|c|c|c|}
\hline Characteristics & $\begin{array}{l}\text { All Groups } \\
(\mathbf{N}=\mathbf{1 0 2})\end{array}$ & $\begin{array}{l}\text { Control } \\
(\mathbf{N}=54)\end{array}$ & $\begin{array}{c}\text { Patient } \\
\text { Advocate } \\
(\text { PA) } \\
(\mathbf{N}=\mathbf{4 8})\end{array}$ \\
\hline \multicolumn{4}{|l|}{ Sociodemographics } \\
\hline $\operatorname{Age}(y)^{*}$ & $51 \pm 13$ & $51.3 \pm 14.3$ & $50.6 \pm 11.0$ \\
\hline Female & $74(72 \%)$ & $34(63 \%)$ & $40(83 \%)$ \\
\hline \multicolumn{4}{|l|}{ Race } \\
\hline Black/African-American & $68(67 \%)$ & $33(61 \%)$ & $35(73 \%)$ \\
\hline White & $24(23 \%)$ & $15(28 \%)$ & $9(19 \%)$ \\
\hline Asian & $1(1 \%)$ & $0(0 \%)$ & $1(2.1 \%)$ \\
\hline No response or declined to answer & $9(9 \%)$ & $6(11 \%)$ & $3(6 \%)$ \\
\hline Ethnicity: Hispanic/Latino & $10(10 \%)$ & $7(13 \%)$ & $3(6 \%)$ \\
\hline \multicolumn{4}{|l|}{ Household income per year } \\
\hline$<\$ 30,000$ & $63(62 \%)$ & $32(59 \%)$ & $31(65 \%)$ \\
\hline$\$ 30,000-\$ 49,999$ & $14(14 \%)$ & $5(9 \%)$ & $9(19 \%)$ \\
\hline$\$ 50,000-\$ 99,999$ & $14(14 \%)$ & $10(19 \%)$ & $4(8 \%)$ \\
\hline$\$ 100,000$ or more & $5(5 \%)$ & $3(6 \%)$ & $2(4 \%)$ \\
\hline Declined to answer & $6(6 \%)$ & $4(8 \%)$ & $2(4 \%)$ \\
\hline \multicolumn{4}{|l|}{ Educational attainment (highest level achieved) } \\
\hline $8^{\text {th }}$ grade or less & $1(1 \%)$ & $0(0 \%)$ & $1(2.1 \%)$ \\
\hline Some high school & $14(14 \%)$ & $2(4 \%)$ & $12(25 \%)$ \\
\hline High school graduate & $40(39 \%)$ & $22(41 \%)$ & $18(38 \%)$ \\
\hline Some college or trade school & $18(18 \%)$ & $12(22 \%)$ & $6(13 \%)$ \\
\hline College graduate & $29(29 \%)$ & $18(33 \%)$ & $11(23 \%)$ \\
\hline \multicolumn{4}{|l|}{ Asthma severity } \\
\hline FEV1 at enrollment (percent predicted) ${ }^{*}$ & $68 \pm 19$ & $68 \pm 20$ & $67 \pm 18$ \\
\hline No. with $\geq 1$ ED visit for asthma in past year & $47(46 \%)$ & $29(54 \%)$ & $18(38 \%)$ \\
\hline No. with $\geq 1$ hospitalization for asthma in past year & $28(28 \%)$ & $18(33 \%)$ & $10(21 \%)$ \\
\hline Asthma-related quality of life ${ }^{* \dagger}$ & $3.9 \pm 1.6$ & $3.9 \pm 1.6$ & $4.0 \pm 1.5$ \\
\hline Asthma control ${ }^{* \dagger \dagger}$ & $2.4 \pm 1.3$ & $2.3 \pm 1.3$ & $2.5 \pm 1.3$ \\
\hline \multicolumn{4}{|l|}{ Literacy } \\
\hline Numeracy $* \mathcal{\xi}$ & $1.9 \pm 1.3$ & $2.2 \pm 1.4$ & $1.7 \pm 1.3$ \\
\hline ANQ $\leq 2$ & $64(63 \%)$ & $29(54 \%)$ & $35(73 \%)$ \\
\hline Reading Comprehension ${ }^{*}, \mathcal{B} \mathcal{B}$ & $28 \pm 11$ & $28 \pm 11$ & $27 \pm 11$ \\
\hline S-TOHFLA $<23$ & $27(26 \%)$ & $14(26 \%)$ & $13(27 \%)$ \\
\hline \multicolumn{4}{|l|}{ Co-morbidities (\#) } \\
\hline Hypertension & $55(54 \%)$ & $31(57 \%)$ & $24(50 \%)$ \\
\hline
\end{tabular}

J Allergy Clin Immunol Pract. Author manuscript; available in PMC 2018 November 01. 


\begin{tabular}{|l|c|c|c|}
\hline Characteristics & $\begin{array}{c}\text { All Groups } \\
(\mathbf{N}=\mathbf{1 0 2})\end{array}$ & $\begin{array}{c}\text { Control } \\
(\mathbf{N}=\mathbf{5 4})\end{array}$ & $\begin{array}{c}\text { Patient } \\
\text { Advocate } \\
(\mathbf{P A}) \\
(\mathbf{N}=\mathbf{4 8})\end{array}$ \\
\hline Diabetes & $25(25 \%)$ & $15(28 \%)$ & $10(21 \%)$ \\
\hline Current smoker & $18(18 \%)$ & $11(20 \%)$ & $7(15 \%)$ \\
\hline Ever smoked & $53(52 \%)$ & $25(46 \%)$ & $28(58 \%)$ \\
\hline
\end{tabular}

mean \pm standard deviation

${ }^{\dagger}$ Asthma-related quality of life was measured with the Mini-Asthma Quality of Life Questionnaire (MiniAQLQ). It is a 15-item questionnaire reflecting well-being over the past 2. It has a 7-point response scale for each item ranging from 1 (maximum impairment) to 7 (no impairment). The average of the items' scores yields the mean summary score. $22-24$

${ }^{\dagger+}$ Asthma control was assessed by the 7-item version of the Asthma Control Questionnaire (ACQ). ${ }^{25-27}$ The score is the mean of all responses $\left(0=\right.$ total control, $6=$ extremely uncontrolled). A score of more than 1.5 is considered inadequate control. ${ }^{28}$

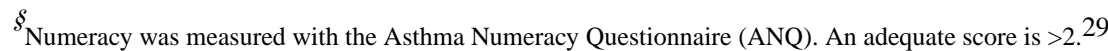

${ }^{\mathcal{B}}$ Reading Comprehension was measured using the Short Test of Functional Health Literacy in Adults (S-TOFHLA). 30 An adequate score is $\geq=23$. 